produire ensuite des souris $\mathrm{XO}$ Sxr', porteuses de la mutation qui fait disparaître H-Y. Leur spermatogenèse est beaucoup plus sévèrement atteinte que celle des XO Sxr, et ne dépasse pas le stade de la spermatogonie. Les auteurs rappellent en outre qu'une souris mâle $\mathrm{XO}$, à spermatogenèse déficiente et dépourvue d'antigène $\mathrm{H}-\mathrm{Y}$, avait été décrite en 1977 et était restée unique.

Peut-on appliquer ces données à l'espèce humaine? Les hommes XX (voir médecine/sciences $n^{\circ} 6$, vol. I, p. 33I) ont, dans la plupart des cas, acquis le facteur masculinisant par transfert de séquences provenant du bras court du chromosome Y. On a décrit un gène de spermatogenèse chez l'homme, probablement homologue de celui de la souris; il est porté par le bras long de $\mathrm{Y}$ et ne devrait donc pas être transféré chez les hommes XX. Or, la recherche de l'antigène $\mathrm{H}-\mathrm{Y}$ s'est montrée négative chez les cing sujets mâles XX qui ont été testés jusqu'à présent. Ces résultats confirment l'hypothèse que, si l'antigène $\mathrm{H}-\mathrm{Y}$ n'est pas le déterminant primaire du sexe, il est un agent indispensable à la spermatogenèse.

J.-C. D.

I. McLaren A, Simpson E, Tomonari $K$, Chandler $P$, Hogg $H$. Male sexual differentiation in mice lacking $\mathrm{H}-\mathrm{Y}$ antigen. Nature $1984 ; 312$ : 552-5.

2. Burgoyne PS, Levy ER, McLaren A. Spermatogenic failure in male mice lacking $\mathrm{H}-\mathrm{Y}$ antigen. Nature 1986; 320: 170-2.

I. Ruoslahti E, Pierschbacher MD. Arg-Gly-Asp: a versatile cell recognition signal. Cell 1986; 44: 517-8.

2. Jenne D, Stanley K. Molecular cloning of S-protein, a link between complement, coagulation and cell-substrate adhesion. EMBO 7 1985; 4: 3153-7.

3. Auffrey C. Un modèle moléculaire de l'interaction entre l'antigène $T_{4}$ et les antigènes HLA de classe II ou le virus LAV. $C R$ Acad Sci Paris 1986; 302: 287-92.

4. Shirakami A, Shigekiyo T, Hirai Y, et al. Plasma fibronectin deficiency in eight members of one family. Lancet 1986; i: 473-4.

\section{Un signal polyvalent de reconnaissance cellulaire}

Un article de Ruoslahti et Pierschbacher (La Jolla, Californie) paru dans Cell [I] passe en revue les propriétés surprenantes d'une séquence de trois acides aminés dans les réactions de reconnaissance entre des protéines qui la contiennent et la surface des cellules. Le modèle de ces protéines est la fibronectine*, glycoprotéine de grande taille, dont le site d'attachement a été isolé, séquencé, et reproduit par synthèse chimique. En raccourcissant progressivement ces peptides de synthèse, on voit qu'au sein de cette protéine de 2500 acides aminés une séquence de trois acides aminés suffit comme signal de reconnaissance. Ce tripeptide, arginine-glycocolle-acide aspartique ou $\mathrm{RGD}^{* *}$ ne peut être modifié sans perdre ses fonctions. Les peptides de synthèse contenant RGD se fixent directement aux cellules lorsqu'ils sont insolubles. S'ils sont solubles, ils inhibent la liaison de la fibronectine aux cellules.

La fibronectine n'est pas restée seule à posséder de telles propriétés. Le fibrinogène contient deux séquences RGD et le facteur Willebrand une, et leur fixation aux plaquettes est empêchée par les peptides de synthèse à RGD. Une autre protéine munie de cette séquence est la vitronectine, le composant actif du facteur d'étalement du sérum. La vitronectine est identique à la protéine $S$, qui forme des complexes avec certains composants du complément et semble également un précurseur de la somatomédine B [2]. Les séquences RGD présentes dans les protéines adhésives sont reconnues par des récepteurs à la surface des cellules. Trois types en ont été identifiés jusqu'à présent. Les deux premiers ont été isolés de cellules d'ostéosarcome humain et de fibroblastes de rat. Ils sont formés chacun de deux chaînes, de taille supérieure à 100000 daltons. L'un est spécifique de la fibronectine, l'autre de la vitronectine. Le troisième, extrait des plaquettes sanguines, présente une spécificité moins étroite, puisqu'il peut se lier à fibronectine, vitronectine, fibrinogène et facteur Willebrand. D'autres récepteurs sont en cours d'isolement, capables notamment de lier le collagène et certains facteurs de croissance.

L'existence de récepteurs différents montre que, si la séquence RGD est nécessaire, elle ne suffit pas à la fixation de toute molécule qui la contient. Deux explications sont proposées. Le tripeptide pourrait être nécessaire comme point d'ancrage, mais la fixation réclamerait un autre site de liaison, spécifique de chaque protéine. Dans l'autre hypothèse, l'information contenue dans RGD serait suffisante, mais les séquences qui l'entourent lui imprimeraient une conformation qui ne s'adapte qu'à certains récepteurs. Les conséquences de cette découverte sont considérables. Dans leur généralité d'abord, car on a trouvé le tripeptide dans des protéines du colibacille et d'une amibe, Dict yostelium discoideum. Pour la différenciation ensuite, au cours de laquelle la capacité de nombreuses cellules à se lier avec des molécules de la matrice varie. Le système de reconnaissance RGD pourrait donc servir de signal de reconnaissance à usages multiples.

Ajoutons enfin qu'en France Charles Auffrey [3] vient de montrer l'importance d'un analogue du RGD où le glycocolle est remplacé par une phénylalanine. Il est présent notamment dans les chaînes $\beta$ des antigènes HLA de classe II, ainsi que dans la protéine $F$ du virus du SIDA, et pourrait jouer un rôle dans l'interaction du virus $\mathrm{LAV}$, comme des antigènes HLA, avec l'antigène $\mathrm{T}_{4}$.

J.-C.D.

* La fibronectine est présente dans le plasma à un taux de 300 à $400 \mathrm{mg}$ par litre. Signalons à cette occasion qu'un déficit familial en fibronectine vient d'être décrit pour la première fois (au fapon) [4]

** Il existe deux nomenclatures pour désigner les acides aminés, une usuelle à trois lettres, une courte à une seule lettre : $A r g=R ; G l y=G ; A s p=D$.
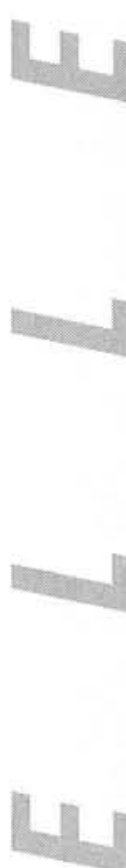